\title{
OPEN Eribulin as a first-line treatment for soft tissue sarcoma patients with contraindications for doxorubicin
}

\author{
Kenji Tsuchihashi ${ }^{1}$, Hitoshi Kusaba ${ }^{1}$, Tomoyasu Yoshihiro ${ }^{2}$, Toshifumi Fujiwara ${ }^{3}$, \\ Nokitaka Setsu ${ }^{3}$, Makoto Endo ${ }^{3}$, Yoshihiro Matsumoto ${ }^{3}$, Takashi Imajima ${ }^{1}$, Yudai Shinohara ${ }^{1}$, \\ Mamoru Ito ${ }^{1}$, Satoru Yamaga ${ }^{2}$, Kenro Tanoue ${ }^{2}$, Kohei Arimizu ${ }^{2}$, Hirofumi Ohmura², \\ Fumiyasu Hanamura ${ }^{2}$, Kyoko Yamaguchi ${ }^{2}$, Taichi Isobe ${ }^{4}$, Hiroshi Ariyama ${ }^{1}$, \\ Yasuharu Nakashima ${ }^{3}$, Koichi Akashi $^{2} \&$ Eishi Baba ${ }^{4 凶}$
}

Doxorubicin is a first-line therapy for patients with unresectable advanced soft tissue sarcoma (STS). However, because of cardiotoxicities, it is not used for patients with cardiac problems. Eribulin has exhibited efficacy for advanced STS in second- or later-line treatments. In the present study, we retrospectively analyzed the efficacy and safety of first-line eribulin therapy for patients with advanced STS unable to receive doxorubicin. Six of 28 patients who received eribulin as any line treatment received eribulin as a first-line treatment. The reasons for avoiding doxorubicin were as follows: cardiac problems for four patients and advanced age for two. Median progression-free survival (PFS) of the patients who received eribulin as first-line and, second or later-line therapy were 9.7 months ( $95 \% \mathrm{Cl}$ : 1.0 -not reached) and 3.9 months $(95 \% \mathrm{Cl}: 2.7-5.9)$, which were not significantly different. The reasons for discontinuation of eribulin were disease progression and adverse events (2 fatigue and 1 neuropathy) for three patients each. No treatment-related cardiotoxicity was observed. The findings of this study indicated that eribulin exhibits meaningful efficacy for the patients with contraindications for doxorubicin as a first-line treatment without cardiac adverse events. However, appropriate safety management is necessary because older patients are typically among those intolerable of doxorubicin.

Soft tissue sarcomas (STS) are heterogenous tumors with over 50 subtypes ${ }^{1}$. Doxorubicin is globally applied as a first-line therapy for patients with unresectable advanced STS. However, doxorubicin has a side effect of cardiotoxicity and is generally contraindicated for patients with current or previous abnormal cardiac function ${ }^{2}$. Clinical trials on doxorubicin have excluded patients with abnormal left ventricular or cardiac ejection fraction ${ }^{3,4}$. STS is common in older patients, and the proportion of patients with STS aged $>60$ years exceeds 50\% in Japan ${ }^{5,6}$. Older patients have an increased risk of doxorubicin-induced heart failure ${ }^{7}$, and old age is reported to be associated with increased hematological toxicity by anthracyclines ${ }^{8}$. Accordingly, the use of doxorubicin tends to be avoided in older populations. Thus, the treatment strategy for the patients with STS with cardiac comorbidities or the aged remains unclear, and is therefore an important issue.

Other chemotherapies for advanced STS are histology-driven, and the application of each drug differs slightly between countries. In Japan, eribulin, pazopanib, trabectedin and ifosfamide are applied ${ }^{1,9-12}$. Eribulin is a microtubule inhibitor that inhibits the growth of microtubules, causing G2/M cell cycle arrest. Eribulin significantly prolonged overall survival (OS) in previously treated patients with advanced liposarcoma (L-sarcoma) or leiomyosarcoma (LMS) compared with dacarbazine in a phase III trial. A phase II trial in Japan also showed the efficacy of eribulin for patients with advanced STS ${ }^{9,12}$. In Japan, eribulin is approved for any kinds of soft tissue

\footnotetext{
${ }^{1}$ Department of Hematology, Oncology and Cardiovascular Medicine, Kyushu University Hospital, Fukuoka 812-8582, Japan. ${ }^{2}$ Department of Medicine and Biosystemic Science, Graduate School of Medical Sciences, Kyushu University, Fukuoka 812-8582, Japan. ${ }^{3}$ Department of Orthopaedic Surgery, Graduate School of Medical Sciences, Kyushu University, Fukuoka 812-8582, Japan. ${ }^{4}$ Department of Oncology and Social Medicine, Graduate School of Medical Sciences, Kyushu University, Fukuoka 812-8582, Japan. ${ }^{\square}$ email: e-baba@ intmed1.med.kyushu-u.ac.jp
} 
sarcoma. On the other hand, for example, eribulin is approved only for liposarcoma in the U.S. based on the data preplanned, exploratory subgroup analyses of OS. Eribulin has been also applied for patients with metastatic breast cancer and its safety and efficacy have been confirmed for older patients ${ }^{13}$.

In the present study, we retrospectively investigated the safety and efficacy of first-line eribulin in patients with STS who were unable to receive doxorubicin because of cardiac comorbidities or advanced age.

\section{Patients and methods}

Patients. We collected data from 28 patients who started eribulin treatment between April 2016 and December 2018 at our institution. The cutoff date was November 2019. Six of 28 patients who received eribulin as any line treatment received eribulin as a first-line treatment. The eligibility criteria were age $\geq 20$ years, histologically proven metastatic or recurrent STS, and receiving eribulin and having sufficient organ function tolerable to chemotherapy.

Treatments. All patients received $1.4 \mathrm{mg} / \mathrm{m}^{2}$ eribulin as a 5-min intravenous infusion on days 1 and 8 every 3 weeks. Initial dose reduction was allowed according to patient status at the discretion of the investigators. Outpatients generally visited the investigators on days 1 and 8 every 3 weeks. At each visit, physical examinations, laboratory tests, and assessments for adverse events (AEs) were performed. The treatment was continued until disease progression, unacceptable toxicity, or a decision to discontinue by the patient or investigator. Dose reduction and treatment delay were performed according to the manufacturer's instructions.

Assessment. Medical information of each patient was retrospectively obtained by electronic records. Tumor lesions were assessed by computed tomography (CT) every 2-3 months. In cases of worsening symptoms or laboratory findings, CT was performed. Progression-free survival (PFS) and OS were defined as the period from the initiation of eribulin to the day of tumor progression or the day of death from any cause, respectively. Tumor responses were assessed using the Response Evaluation Criteria in Solid Tumors (RECIST) version 1.1. Adverse events during the therapy were evaluated according to the National Cancer Institute Common Terminology Criteria for Adverse Events (CTCAE) version 4.0.

Statistical analysis. PFS and OS of two specific patients groups were estimated using the Kaplan-Meier method, compared wth the log-rank test. Other comparisons of two specific patient groups were performed using chi-squared test and Fisher's exact test. Hazard ratios were calculated using a Cox proportional hazard model. Values of $p<0.05$ were considered significant. Relative dose intensity was the percentage of actual cumulative dose compared with the amount of planned cumulative dose. All statistical analyses were carried out using JMP software (SAS Institute Japan, Tokyo, Japan).

Ethics approval. All procedures performed in studies involving human participants were in accordance with the ethical standards of the institutional and/or national research committee and with the 1964 Helsinki declaration and its later amendments or comparable ethical standards. This study was approved by the Ethics Committee of Kyushu University Hospital (Approval No. 2019-618).

Informed consent. Informed consent was not obtained from each patient due to the retrospective nature of the present study. The consent was waived by the Ethics Committee of Kyushu University Hospital.

\section{Results}

Patients' characteristics. A total of 28 patients with STS were analyzed. Six and twenty-two patients received eribulin as a first-line treatment and, a second or later-line treatment, respectively. The patients' characteristics are shown in Table 1 . The median age of the patients who received eribulin as a first-line therapy was 76 years (range: 58-82 years), which is significantly older than 62 years (range: $20-76$ years) for the patients who received eribulin as a second or later-line therapy. Five patients (83\%) were male. Performance status 0 or 1 was observed in three patients (50\%). Histology was L-sarcoma, LMS, and others for two patients each. The reason for avoiding doxorubicin therapy was cardiac problems for four patients and advanced age for two.

Treatment and efficacy. The median follow-up time was 16 months for patients who received first-line eribulin therapy. At the data cutoff, no patients received eribulin. The reasons for discontinuation of eribulin were disease progression and adverse events ( 2 fatigue and 1 neuropathy) for three patients each. The median number of cycles of eribulin treatment was 7.5 (range: 2-15). The median relative dose intensity was $93 \%$ (range: 49-99\%). The reasons for cessation of eribulin treatment were progressive disease and AEs in $50 \%$ of the patients each. These AEs were two cases of fatigue and one of neuropathy. Median PFS in the six patients treated with eribulin as a first-line treatment was 9.7 months (95\% confidence interval [CI]: 1.0 month-not reached) (Fig. 1a). The PFS rate at 12 weeks was $67 \%$. OS was 39.4 months (95\% CI: 1.7-39.4 months) (Fig. 1b). The response rate for the five patients with measurable lesions was $0 \%$, and the disease control rate was $66.7 \%$. On the other hand, the median number of cycles of eribulin treatment for the patients who received second or later-line eribulin therapy was 7.7 (range: 2-22). The median relative dose intensity was $79 \%$ (range: $31-100 \%$ ). The reasons for cessation of eribulin treatment were progressive and surgery in $91 \%$ and $9.0 \%$ of the patients each. Median PFS was 3.9 months (95\% CI: 2.7-5.9 months) (Fig. 1a). The PFS rate at 12 weeks was $65 \%$ for twenty patients except for two patients who received surgery. Within twenty-two patients who received eribulin as a second or later-line treatment, twelve patients were initially diagnosed as unresectable or metastatic and received first-line chemo- 


\begin{tabular}{|c|c|c|c|c|}
\hline & Total $(\mathbf{n}=28)$ & First line $(n=6)$ & Second or later line $(n=22)$ & $p$ value \\
\hline Median age, years (range) & $67(20-82)$ & $76(58-82)$ & $62(20-76)$ & 0.013 \\
\hline Sex, n (\%) & & & & 0.16 \\
\hline Male & $14(50)$ & $5(83)$ & $9(32)$ & \\
\hline Female & $14(50)$ & $1(17)$ & $13(68)$ & \\
\hline ECOG PS, n (\%) & & & & 0.40 \\
\hline 0 & $2(7)$ & $1(17)$ & $1(5)$ & \\
\hline 1 & $13(46)$ & $2(33)$ & $11(50)$ & \\
\hline 2 & $11(39)$ & $2(33)$ & $9(41)$ & \\
\hline 3 & $2(7)$ & $1(17)$ & $1(5)$ & \\
\hline Histology, n (\%) & & & & 0.72 \\
\hline Dedifferentiated liposarcoma & 10 & $2(33)$ & $8(29)$ & \\
\hline Leiomyosacroma & 6 & $2(33)$ & $4(14)$ & \\
\hline Others & 12 & $2(33)$ & $10(38)$ & \\
\hline \multicolumn{5}{|l|}{ Previous chemotherapy, n (\%) } \\
\hline 0 & $6(21)$ & $6(100)$ & 0 & \\
\hline 1 & $9(32)$ & 0 & $9(41)$ & \\
\hline$>1$ & $13(47)$ & 0 & $13(59)$ & \\
\hline \multicolumn{5}{|c|}{ The reason for avoiding doxorubicin } \\
\hline Cardiac comorbidities & $4(14)$ & 4 & 0 & \\
\hline Old age & $2(7)$ & 2 & 0 & \\
\hline
\end{tabular}

Table 1. Patient's characteristics. ECOG PS Eastern Cooperative Oncology Group Performance Status.

a

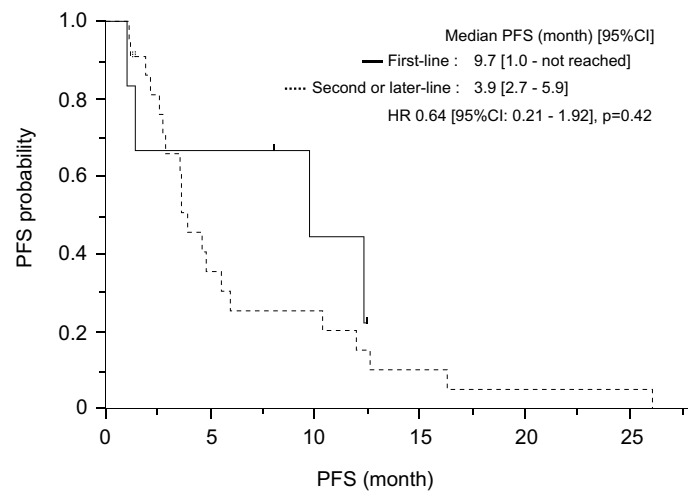

b

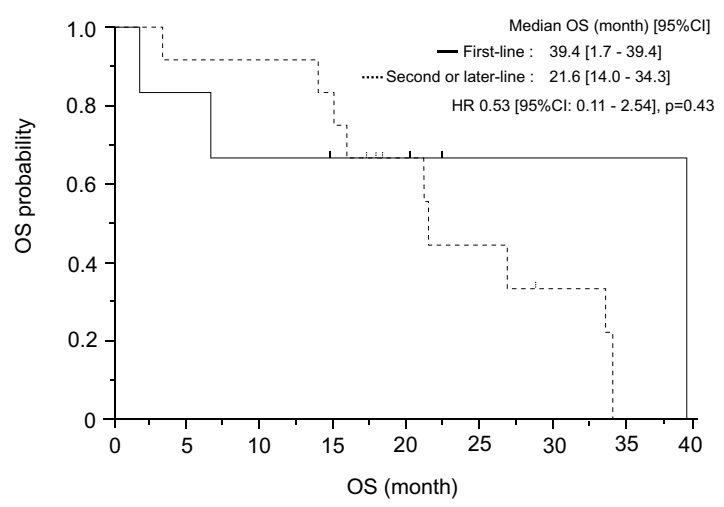

Figure 1. (a) Kaplan-Meier plot for progression-free survival (PFS) of patients who received eribulin as a firstline therapy, and second or later-line therapy. 95\% CI, confidence interval. HR, hazard ratio. (b) Kaplan-Meier plot for overall survival (OS) of patients who received eribulin as a first-line therapy, and second or later-line therapy. 95\% CI, confidence interval. HR, hazard ratio. 


\begin{tabular}{|c|c|c|c|c|c|c|}
\hline & \multicolumn{3}{|l|}{ First line } & \multicolumn{3}{|c|}{ Second or later line } \\
\hline & All Grade, n (\%) & Grade 3, n (\%) & Grade 4, n (\%) & All Grade, n (\%) & Grade 3, n (\%) & Grade 4, n (\%) \\
\hline Any adverse events & $6(100)$ & $5(83)$ & $2(33)$ & $22(100)$ & $14(64)$ & $10(45)$ \\
\hline Leukopenia & $4(67)$ & 0 & $1(17)$ & $20(91)$ & $3(14)$ & $3(14)$ \\
\hline Neutropenia & $5(83)$ & $2(33)$ & $2(33)$ & $20(91)$ & $3(14)$ & $10(45)$ \\
\hline Lymphopenia & $2(33)$ & 0 & $1(17)$ & $15(68)$ & $3(14)$ & $3(14)$ \\
\hline Anemia & $5(83)$ & 0 & 0 & $20(91)$ & $2(9)$ & 0 \\
\hline Thrombocytopenia & $3(50)$ & $1(17)$ & 0 & $4(18)$ & 0 & 0 \\
\hline Mucositis oral & $2(33)$ & $1(17)$ & 0 & $4(18)$ & 0 & 0 \\
\hline Sore throat & $1(17)$ & 0 & 0 & $2(9)$ & 0 & 0 \\
\hline Hiccups & $1(17)$ & 0 & 0 & 0 & 0 & 0 \\
\hline Constipation & $1(17)$ & 0 & 0 & $6(27)$ & 0 & 0 \\
\hline Diarrhea & $1(17)$ & 0 & 0 & 0 & 0 & 0 \\
\hline Appetite loss & $3(50)$ & 0 & 0 & $10(45)$ & 0 & 0 \\
\hline Fatigue & $4(67)$ & $1(17)$ & 0 & $10(45)$ & 0 & 0 \\
\hline Alopecia & $3(50)$ & 0 & 0 & $3(14)$ & 0 & 0 \\
\hline Limb edema & $1(17)$ & 0 & 0 & $3(14)$ & 0 & 0 \\
\hline Arthralgia & $2(33)$ & 0 & 0 & 0 & 0 & 0 \\
\hline Myalgia & $1(17)$ & 0 & 0 & $1(5)$ & 0 & 0 \\
\hline Dizziness & $1(17)$ & 0 & 0 & $1(5)$ & 0 & 0 \\
\hline Peripheral neuropathy & $4(67)$ & $1(17)$ & 0 & $7(32)$ & 0 & 0 \\
\hline Rash & $2(33)$ & 0 & 0 & $3(14)$ & 0 & 0 \\
\hline Skin (Folliculitis) & $1(17)$ & 0 & 0 & 0 & 0 & 0 \\
\hline Infection & $3(50)$ & $1(17)$ & 0 & $5(23)$ & $1(5)$ & 0 \\
\hline Febrile neutropenia & $1(17)$ & $1(17)$ & 0 & $3(14)$ & $3(14)$ & 0 \\
\hline AST elevation & $4(67)$ & 0 & 0 & $13(59)$ & 0 & 0 \\
\hline ALT elevation & $2(33)$ & 0 & 0 & $7(32)$ & 0 & 0 \\
\hline ALP elevation & $3(50)$ & 0 & 0 & $6(27)$ & 0 & 0 \\
\hline Hypokalemia & 0 & 0 & 0 & $5(23)$ & 0 & 0 \\
\hline
\end{tabular}

Table 2. Summary of Treatment related adverse events. AST aspartate aminotransferase, $A L T$ alanine aminotransferase, $A L P$ alkaline phosphatase.

therapy. OS for those patients from first-line chemotherapy 21.6 months (95\% CI: 14.0-34.3 months) (Fig. 1b). PFS and OS of the patients who received eribulin as a first-line and, second or later-line therapy were not significantly different: HR 0.64 (95\% CI: $0.21-1.92), p=0.42$ for PFS, and HR 0.53 (95\% CI: 0.11-2.54) $p=0.43$.

Safety. All of the patients treated with first-line, and second or later-line eribulin experienced $\geq 1 \mathrm{AE}$, as shown in Table 2. Grade 4 AEs in patients treated with first-line, and second or later-line eribulin included leukopenia (17\% and $14 \%$ ), neutropenia (33\% and $45 \%)$ and lymphopenia (17\% and $14 \%)$. Infection of any grade in patients treated with first-line, and second or later-line eribulin was $50 \%$ and $23 \%$. Febrile neutropenia in patients treated with first-line, and second or later-line eribulin was $17 \%$ and $14 \%$.

\section{Discussion}

The results of the present study indicate the efficacy of eribulin as a first-line therapy for patients with STS who have contraindications for doxorubicin. No cardiac AEs were observed. However, as many patients with contraindications for doxorubicin are aged, careful safety management of eribulin is necessary.

Here, the efficacy of eribulin is reported for patients with STS previously treated with at least one chemotherapy regimen. PFS was 2.6 months (95\% CI: 1.9-2.8) in a phase III trial of previously treated patients ${ }^{9}$. In this trial, $50 \%$ of the patients received two previous chemotherapy regimens and $50 \%$ received more than two regimens before eribulin. In a phase II trial of eribulin for previously treated patients with STS, PFS was 4.1 months (95\% CI: $2.6-5.6)^{14}$. In this trial, the median number of prior chemotherapy regimens was two (range: $1-7$ ). In the present study, PFS of the patients who received eribulin as a first-line therapy was 9.7 months (95\% CI: 1.0-not reached), which is better than that in published reports of previously treated patients. PFS in a phase III trial of doxorubicin as a first-line treatment for advanced or metastatic STS was 4.6 months (95\% CI: 2.9-5.6 months) ${ }^{3}$. A previous report showed that eribulin was especially effective for dedifferentiated liposarcoma (DDLS) ${ }^{15}$. The histology of six patients treated in the present trial were DDLS $(n=2)$, LMS $(n=2)$, myxofibrosarcoma $(n=1)$, and unclassified histology $(n=1)$. PFS of one DDLS and one myxofibrosarcoma patients were censored because of surgery and radiation. PFS of DDLS cases were 12.5 and 12.3 months. PFS of LMS cases were 9.7 and 1.4 months. PFS of myxofibrosarcoma and unclassified histology cases was 8.3 and 1.0 months. Our result was consistent with that of a previous report showing that eribulin had strong potency for DDLS. The response rate for eribulin 
in patients with measurable lesions was $0 \%$. The response rate for eribulin in previous trials with patients previously treated was $0-4 \%^{7,10}$. Our results indicated that the response rate for eribulin was low, even in first-line therapy. PFS of the patients who received eribulin as a second or later-line treatment was 3.9 months (95\% CI: 2.7-5.9 months). This result is almost equivalent to the previous report ${ }^{9}$. Although PFS of the patients who received eribulin as first-line and, second or later-line therapy were not significantly different, the efficacy of eribulin as a first-line therapy is considered as meaningful because the population for a first-line eribulin therapy is advanced age and has cardiac problems, and is considered as contraindication for receiving doxorubicin which is a standard first-line therapy.

The reasons for avoiding doxorubicin were cardiac problems for four patients and advanced age for two. Cardiac problems were one case of cardiac function impairment with multiform premature ventricular contraction, one case of hypertrophic cardiomyopathy, and two cases of myocardial perfusion scintigraphy. In the present study, no cardiac AEs were observed.

In addition, $83 \%$ of the patients who received eribulin as first-line treatment experienced a grade 3 or higher AE. Febrile neutropenia was observed in $17 \%$ of the patients, grade $3 / 4$ neutropenia was observed in $67 \%$ of the patients, and grade 3 neuropathy and fatigue were observed in $17 \%$ of the patients. Previous clinical trials investigating eribulin for STS reported that $96 \%$ of patients experienced a grade 3 or higher AE, including grade $3 / 4$ neutropenia $(86.3 \%)$ and grade 3 febrile neutropenia (8\%), which is comparable with the present trial ${ }^{10}$. However, the rate of any infection in the patients who received eribulin as a first-line treatment was higher than the patients who received eribulin as a second or later-line treatment. Further, $50 \%$ of the patients in the present trial withdrew from treatment because of AEs, which is more frequent than the $8 \%$ reported in past trials. The AEs related to withdrawal were fatigue and neuropathy. The mean age of the participants in the present trial was 76 years (range: $58-82$ years), which was higher than that reported in previous studies (mean: 52 years; range: 28-73 years). This finding suggests that patients with contraindications for doxorubicin tend to be of advanced age, and thus, careful safety management of eribulin is necessary.

The major limitation of the present study is its retrospective design and limited sample size. Further, the approval of eribulin is different between countries. In Japan, eribulin is approved for any kinds of soft tissue sarcoma, but, for example, approved only for liposarcoma in the U.S. Accordingly, the efficacy of eribulin as firstline treatment needs to be more evaluated depending on the approval status of each countries.

In conclusion, the results of the present study demonstrated the safety and efficacy of eribulin for patients inapplicable with contraindications for doxorubicin as a first-line treatment without cardiac AEs. However, careful safety management is necessary because most patients intolerable of doxorubicin are of advanced age.

Received: 8 June 2020; Accepted: 17 November 2020

Published online: 01 December 2020

\section{References}

1. Ratan, R. \& Patel, S. R. Chemotherapy for soft tissue sarcoma. Cancer 122, 2952-2960 (2016).

2. Raj, S., Franco, V. I. \& Lipshultz, S. E. Anthracycline-induced cardiotoxicity: A review of pathophysiology, diagnosis, and treatment. Curr. Treat. Options Cardiovasc. Med. 16, 315 (2014).

3. Judson, I. et al. Doxorubicin alone versus intensified doxorubicin plus ifosfamide for first-line treatment of advanced or metastatic soft-tissue sarcoma: A randomised controlled phase 3 trial. Lancet Oncol. 15, 415-423 (2014).

4. Seddon, B. et al. Gemcitabine and docetaxel versus doxorubicin as first-line treatment in previously untreated advanced unresectable or metastatic soft-tissue sarcomas (GeDDiS): A randomised controlled phase 3 trial. Lancet Oncol. 18, 1397-1410 (2017).

5. Ogura, K., Higashi, T. \& Kawai, A. Statistics of soft-tissue sarcoma in Japan: Report from the Bone and Soft Tissue Tumor Registry in Japan. J. Orthop. Sci. 22, 755-764 (2017).

6. Younger, E. et al. Outcomes of elderly patients with advanced soft tissue sarcoma treated with first-line chemotherapy: A pooled analysis of 12 EORTC soft tissue and bone sarcoma group trials. Oncologist 23, 1250-1259 (2018).

7. Accordino, M. K., Neugut, A. I. \& Hershman, D. L. Cardiac effects of anticancer therapy in the elderly. J. Clin. Oncol. 32, 2654-2661 (2014).

8. Garbay, D. et al. Advanced soft-tissue sarcoma in elderly patients: Patterns of care and survival. Ann. Oncol. 24, 1924-1930 (2013).

9. Schöffski, P. et al. Eribulin versus dacarbazine in previously treated patients with advanced liposarcoma or leiomyosarcoma: A randomised, open-label, multicentre, phase 3 trial. Lancet 387, 1629-1637 (2016).

10. van der Graaf, W. T. A. et al. Pazopanib for metastatic soft-tissue sarcoma (PALETTE): A randomised, double-blind, placebocontrolled phase 3 trial. Lancet 379, 1879-1886 (2012).

11. Demetri, G. D. et al. Efficacy and safety of trabectedin or dacarbazine for metastatic liposarcoma or leiomyosarcoma after failure of conventional chemotherapy: Results of a phase III randomized multicenter clinical trial. J. Clin. Oncol. 34, 786 (2016).

12. Kawai, A., Yonemori, K., Takahashi, S., Araki, N. \& Ueda, T. Systemic therapy for soft tissue sarcoma: Proposals for the optimal use of pazopanib, trabectedin, and eribulin. Adv. Ther. 34, 1556-1571 (2017).

13. de Nonneville, A. et al. Safety and efficacy of eribulin for 'real-world' older patients with metastatic breast cancer. J. Geriatr. Oncol. 9, 281-283 (2018).

14. Kawai, A. et al. Phase 2 study of eribulin in patients with previously treated advanced or metastatic soft tissue sarcoma. Jpn. J. Clin. Oncol. 47, 137-144 (2017).

15. Demetri, G. D. et al. Activity of eribulin in patients with advanced liposarcoma demonstrated in a subgroup analysis from a randomized phase III study of eribulin versus dacarbazine. J. Clin. Oncol. 35, 3433-3439 (2017).

\section{Author contributions}

Conception and design: Kenji T., H.K., Y.M., E.B. Acquisition of data: Kenji T., T.Y., T.F., N.S., M.E., S.Y., Kenro T., Kohei A., H.O. Analysis and interpretation of data: Kenji T., T.Y., T.F., N.S., M.E., T.I., Y.S., M.I., S.Y., Kenro T., Kohei A., H.O., F.H., K.Y., T.I., H.A. Writing, review, and/or revision of the manuscript: Kenji T., H.K., Y.M., Y.N., Kohei A., E.B. 


\section{Funding}

This research is funded by Japan Society for the Promotion of Science KAKENHI grant (K.T.).

\section{Competing interests}

K.T. received honoraria from Eisai, Chugai Pharma and Novartis Pharma. N.S. received honoraria from Daiichi Sankyo. M.E. received honoraria from Eisai, Taisho, Daiichi Sankyo, Novartis Pharma and Taisho-Toyama. M.E. received consulting fee from Eli Lilly. Y.M. received honoraria from Eisai, Taiho Pharma, Synthes and Medtronic. M.T. received honoraria from Chugai Pharma and Japan Blood Product Organization. H.A. received honoraria from Chugai Pharma. Y.N. received honoraria from Chugai Pharmaceutical, Mitsubishi-Tanabe Pharma, Janssen Pharmaceutical, Pfizer, Eisai, AbbVie, Bristol-Myers Squibb, Astellas, AYUMI, Hisamitsu, Taisho Pharmaceutical, Takeda Pharmaceutical, Zimmer-Biomet and Kyocera. Y.N. received donation from Kyocera. K.A. received honoraria from Astellas Pharm, AbbVie GK, Eisai, Kyowa Kirin, Novartis Pharma, Bristol-Myers Squibb, Celgene, Janssen Pharma, Takeda Pharma, Chugai Pharma, Otsuka Pharma, Daiichi Sankyo, Amgen Astellas BioPharma and Ono Pharma. K.A. received donation from Astellas Pharma, Eisai, Sanofi, Asahi Kasei Pharma, Japan Blood Products Organization, Kyowa Kirin, Mochida Pharma, Ono Pharma, Otsuka Pharma, Shionogi, Yakult Honsh, Sumitomo Dainippon Pharma,Taiho Pharma, Daiichi Sankyo, Chugai Pharma, Nippon Kayaku, Nippon Shinyaku, Nihon Pharma, FUJIFILM Toyama Chemical, Takeda Pharma, Novartis Pharma, MSD and Eli Lilly Japan. E.B. received honoraria from Eli Lilly Japan. No other authors have no competing interests.

\section{Additional information}

Correspondence and requests for materials should be addressed to E.B.

Reprints and permissions information is available at www.nature.com/reprints.

Publisher's note Springer Nature remains neutral with regard to jurisdictional claims in published maps and institutional affiliations.

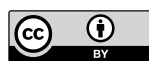

Open Access This article is licensed under a Creative Commons Attribution 4.0 International License, which permits use, sharing, adaptation, distribution and reproduction in any medium or format, as long as you give appropriate credit to the original author(s) and the source, provide a link to the Creative Commons licence, and indicate if changes were made. The images or other third party material in this article are included in the article's Creative Commons licence, unless indicated otherwise in a credit line to the material. If material is not included in the article's Creative Commons licence and your intended use is not permitted by statutory regulation or exceeds the permitted use, you will need to obtain permission directly from the copyright holder. To view a copy of this licence, visit http://creativecommons.org/licenses/by/4.0/.

(C) The Author(s) 2020 